\title{
A method of enclosing a target by robot swarm with limited range homogeneous signal emitter
}

\author{
Masao Kubo \\ Computer Science, National Defense Academy of Japan, Yokosuka, Kanagawa, Japan \\ Hiroshi Sato \\ Computer Science, National Defense Academy of Japan, Yokosuka, Kanagawa, Japan
}

Akihiro Yamaguchi

Department of Information and Systems Engineering, Fukuoka Institute of Technology Fukuoka, Japan

\author{
Akira Namatame \\ Computer Science, National Defense Academy of Japan, Yokosuka, Kanagawa, Japan \\ Eugene Kitamura \\ Computer Science, National Defense Academy of Japan, Yokosuka, Kanagawa, Japan \\ E-mail: \{masaok,hsato,nama,ed12005\}@nda.ac.jp,aki@fit.ac.jp
}

\begin{abstract}
This paper presents a robot swarm model to enclose a target. The robots use information of the target and each of their neighbors. Every robot has its own, standard torch-like signal emitter and they can observe the sum of the intensity of their torches. In this paper, the robots identify their nearest neighbor by choosing the emitting source with the strongest intensity. We expect that this new approach makes the robot swarm more simple and scalable. We confirm this model by computer simulations.
\end{abstract}

Keywords: target enclosure, vehicle formation, collective motion, swarm robotics

\section{Introduction}

In this paper, a new implementation of a robot swarm for enclosing a target is examined. The swarm is equipped with the same limited transmission range signal emitter. This communication system is believed to simplify the building of a large swarm.
We focused on the study of Takayama et al. ${ }^{7}$. In this model, each robot needs information of directions to one neighbor and to its target. As in other studies, this model also requires the Hamiltonian cycle constraint ${ }^{7}{ }^{4}$. Recently, research used a hybrid system theory ${ }^{5}$ and showed that robots controlled by Takayama's method which references the nearest neighbor can also enclose a target. The robot swarm with this new reference 
model can enclose targets while the size of the group is changing 3 .

Scalability is one of the important properties of a robotic swarm. The target enclosing algorithm proposed in this paper has this scalability, the accurate observation of the neighborhood usually becomes difficult as the number of robots increases.

Various ways to know who is the nearest neighboring robot can be enumerated as follows. (1) Every robot has its unique signal emitter and a receiver measures the power of the incoming signals. (2) Each robot observes its neighborhood visually and measures the size of the robots come in sight. These 2 methods are based on relative distance among robots. (3) Each robot calculates its own location on a common reference frame. The information of the robot's location is exchanged by broadcasting. The first method is stable but the number of robot is limited. The second method is reasonable but the visibility is strongly influenced by environmental conditions. The third method is the best way when we can use GPS and a wireless communication network.

We focus on a system with limited range homogeneous signal emitter. We call it the torch system. For example, Kilobot ${ }^{6}$ has the same small LED emitter as a communication device and a flock of 1000 Kilobots can form a large shape. However, the transmission range of their LED light is about 6 robots length. Swarmbot ${ }^{9}$ proposes that the robot having a homogeneous light can collect the sufficient number of robots to solve a task. Each robot has the color lights around its body. The intensity of light becomes strong as the number of robots increase. If the intensity of light is too strong a robot will not join the group.
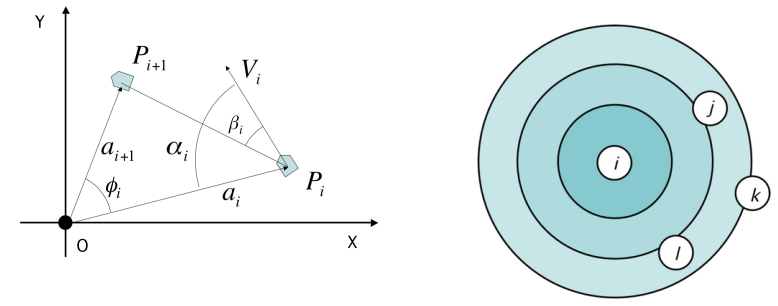

Fig.1 Model of Takyama's Fig.2 Diffusion of light algorithm: $\alpha_{i}, \beta_{i}$.
The advantage of this torch system is its high procurability. The very limited transmission range provides less interference of communication. Therefore, emitters which use the same signal band can be installed into many robots. Also, its small energy consumption is an advantage. The smaller the communication range, the smaller the energy consumption.

In this paper, we examine a robotic swarm with torch system for the target enclosing task. We adopt Takayama's work for the control scheme of robot but the referencing robot is different in this work. We propose the most powerful signal direction to be the direction of the referencing robot. We call this the "MOPS" direction. In this paper, we show that the proposed robotic swarm can enclose a target successfully by computer simulation.

\section{Takayama's target enclosing model}

Firstly, Takayama's target enclosing model is explained. We assume that all robots choose the same target. On a two-dimensional (2D) plane, let there be only one target $O$ at the origin and $n$ robots. Robots are numbered counterclockwise as $P_{1, . .,} P_{n}$, and $r_{i}$ is the position vector of the robot $P_{i}$.

To achieve this task, Takayama et al. ${ }^{7}$ proposed the following model (Fig.1). Each robot determines its control input, speed $v_{i}$, and angular velocity $\omega_{i}$ using two aspects of angular information: relative angles with respect to the target and an anterior neighboring robot, denoted as $\alpha_{i}$ and $\beta_{i}$, respectively. As a result, rotational movement occurs with a central focus on the target.

$$
\begin{aligned}
v_{i} & =f \beta_{i} \\
\omega_{i} & =\frac{v_{i}}{\bar{r}}-k \cos \left(\alpha_{i}\right)
\end{aligned}
$$

where the parameters $\bar{r}, k$, and $f>0$ as specified beforehand. $P_{i+1}$ is the robot to which $P_{i}$ refers, and $\bar{r}$ is the expected distance to the target. In Takayama et al.'s model, the $i$-th robot refers to the $i+1$-th robot, and the $n$-th robot refers to the first robot $P_{1}$. That is, if the relationship between a robot and its reference robot is considered as a link in graph theory, the graph of the group of robots must be a Hamiltonian cycle. The authors proved the convergence to the 
goal state of the target enclosing task under this constraint ${ }^{3}$.

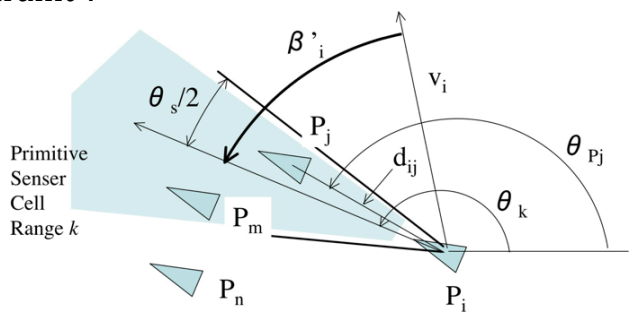

Fig.3 The most powerful signal (MOPS) direction

\section{Nearest neighboring robot as the reference}

Ref.3 examined a new reference robot scheme in which each robot considers its nearest neighboring robot as its reference robot. Each robot controls itself as described in equations 1 and 2 , but it chooses its nearest neighbor as its reference robot. This system has higher scalability because the individual robots need not be identified to observe the nearest robot.

\section{The proposed reference model: the most powerful signal direction reference model, MOPS model}

We propose a new robotic swarm for the target enclosure task. Each robot carries a torch with the same specification. Additionally we propose that the direction of the most powerful signal is used as the direction of the referencing robot.

\section{1. Torch}

Every agent has the same torch and the agent can turn on/off its torch. The maximum intensity of all the torches is the same and known beforehand. We suppose that the speed of propagation of the signal of the torch is so fast that an agent can observe the signal immediately. As shown in Fig.2, during the flight of the signal, the power of the signal is attenuated. The signal propagation function $p(d)$ is described as follows.

$$
p(d)=\left\{\begin{array}{cc}
\gamma^{(d / L)} /(d / L)^{2} & d>L \\
1 & \text { otherwise }
\end{array}\right.
$$

where $d$ is the distance from the emitter, $L$ is the length of an agent, $\gamma$ is the attenuation coefficient per $L$. The equation 3 represents the spherical diffusion of a light. An agent closer to an emitter receives a stronger signal.

\subsection{Target enclosing model with MOPS direction reference model}

We explain the procedure of receiving a signal in the proposed method.

Agent $P_{i}$ uses the most powerful signal direction instead of the angle from $P_{i}$ to $P_{j}\left(=\beta_{i}\right)$. Also, we call the angle $\beta_{i}^{\prime}$ '. Fig.3 shows this angle. Now, we suppose agent $P_{i}$ has several signal receivers. We call it sensor primitive. The direction of $k$-th sensor primitive $s p_{k}$ is $\theta_{k}$. If $\left|\theta_{k^{-}} \theta_{i j}\right| \leqq \theta_{s} / 2$, the sensor primitive $s p_{k}$ can sense the signal of $P_{j}$. We call $\theta_{s}$ a visible range of sensor primitive. The power of sensor primitive $s p_{k}, s_{k}$ is

$$
s_{k}=\sum_{P_{m} \in V i s i b l e} p\left(d_{i, m}\right)
$$

where $d_{i, m}$ is the distance between $P_{m}$ and $P_{i}$ and $p$ is the signal propagation function in eq.3. $\beta_{i}{ }^{\prime}$ is the substitution of $\beta_{i}$.

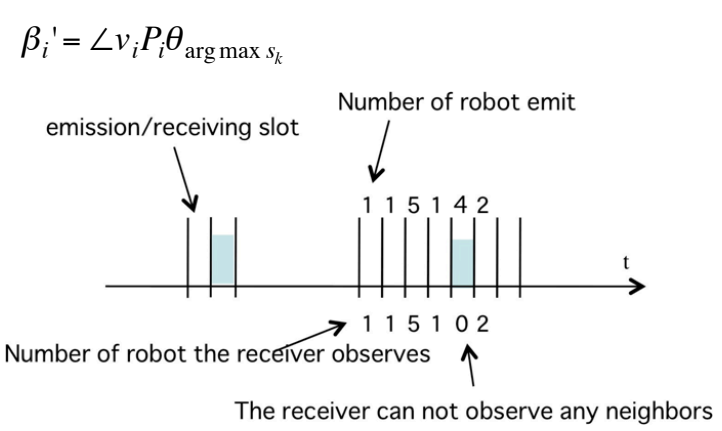

Fig.4 The number of signal which a robot can receive

\section{Torch flashing signal strategy}

In this section, we propose several torch flashing strategies and evaluate them by computer simulation.

We show the high performance of protocol $B$ which can collect sufficient information about neighbor agents.

Generally, an agent cannot observe its neighborhood when its torch is turned on because the power of its own torch is too strong. This is a dilemma. An agent should turn its torch on to notify 
other agents its existence. However, an agent should turn its torch off to know the existence of other agents. Therefore, some reasonable torch flashing signal strategies are required.

We examine the following 5 strategies. (A) Turn on with probability $r$ and stop after the lapse of a fixed predetermined seconds (we call this strategy random_start+fixed_output_span). (B) Turn on when someone starts and stop with probability $r$ (reactive+random_stop). (C) Turn on when someone starts and stops after the lapse of a fixed predetermined seconds (reactive+minimum_span). (D) Turn its torch on always and take a break with fixed timing (greedy_fixed_output_span). (E) Turn its torch on always and take a break with probability $r$ (greedy_random_stop).

The criteria of the performance of protocol is the average of the number of signals recognized per interval on which an agent emits. We call this interval slot. Fig.4 illustrates an example of emission and signal receiving process of an agent. All agents turn their own torches on or off according to the slot. The numbers above the line represent the number of agents which turn their torch on at each slot. If an agent turns its torch off, the agent can collect correct information of these agents. The number of information of agents which the agent can collect is shown below the line. The performance of protocol $x$, eval $_{X}$ is the average of these, namely,

$\operatorname{eval}_{x}=\sum_{i \in N} \frac{\sum_{s \in S} \operatorname{receive}(s, i)}{|S|} /|N|$

$\operatorname{receive}(s, i)=\left\{\begin{array}{cc}0 & i \in \operatorname{Emits}(s) \\ |\operatorname{Emits}(s)| & \text { otherwise }\end{array}\right.$

where $\operatorname{Emit}(s)$ is a set of agents whose torches are on during slot $s$.

Fig. 5 shows the performance of the 5 strategies in the case of a group of 10 agents by computer simulation. The $x$ axis indicates the parameter $r$ and the $y$ axis shows eval . Strategy $B$ is better than the others. This strategy can provide an average of 2.24 agents' information. This suggests that an agent with strategy $B$ can collect information of all agents during 5 slots $>10 / 2.24$.

Fig.6 shows $/ N / /$ eval(B) of strategy $B . / N / /$ eval(B) is the average number of slots which a robot required to collect information of all agents. As this graph indicates, /N//eval(B) is almost flat and it shows that the agent can collect all members' information during the fixed time span despite of the swarm size $/ N /$.

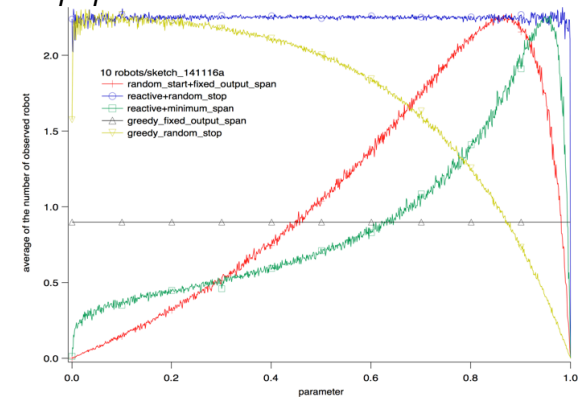

Fig.5 The performance of the 5 proposed strategies $(10$ agents)

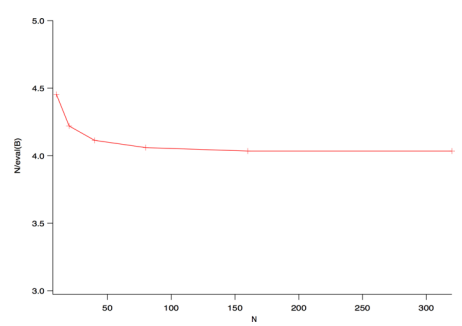

Fig.6 The performance of strategy B according to the number of agents.

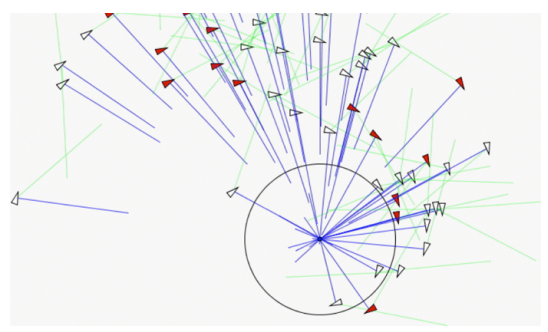

Fig.7 An example of initial state of target enclosure with 60 agents

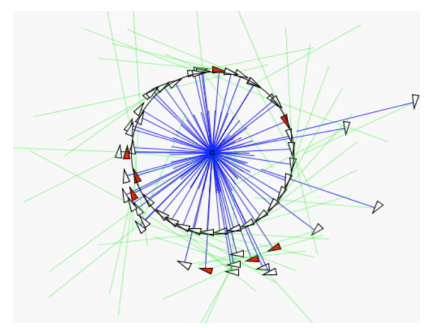

Fig.8 An example of near completion of target 
enclosure with 60 agents

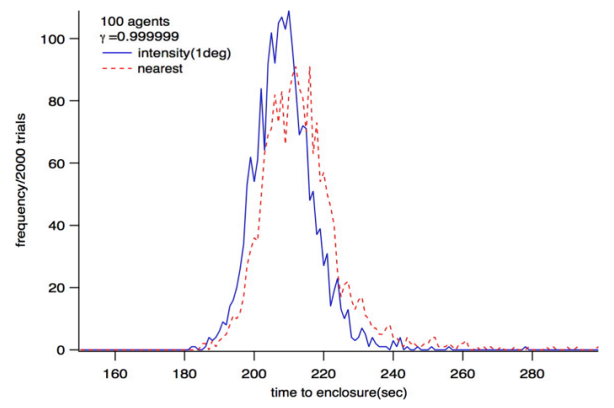

Fig.9 Histogram of time to enclose a target by 100 agents. 2000 trials are examined.

In the above experiment, the agent with strategy $B$ can collect sufficient information to do the target enclosing task in a constant time span. In the next section, we show the result of target enclosing by computer simulation.

\section{Computer simulation}

In this section, the result of computer simulation of enclosing a target by a robot swarm with the proposed MOPS direction reference model (eq.5) is shown.

The setting of experiment is explained. There is 1 target at the origin. There are / $N /$ agents which are deployed at random distances from the origin. We suppose that the agents use strategy $B$ and every agent can receive a torch signal from all agents.

Examples of target enclosing process are shown in Fig.7, Fig.8. In this case, we set $|N|=60$ and the attenuation coefficient $\gamma=0.9999$. The circle indicates the orbit on which agents enclose the target. A triangle indicates an agent. The 2 lines from each triangle represent its target and the direction $\beta_{i}{ }^{\prime}$. If the gap between $\beta_{i}{ }^{\prime}$ and the direction to its nearest neighbor agent is large, namely $\left(\left|\beta_{i}{ }^{\prime}-\beta_{i}\right|\right.$ $>\theta_{s}$ ), the triangle is filled. As you see in Fig.8, the agents can enclose the target and as the number of the agents on the orbit increases, the number of filled triangles decreases.

This attempt to enclose the target was repeated 2000 times. The group size $/ N /$ is 100 . At every attempt the agents started from different positions. All the attempts succeeded. Fig.9 shows the histogram of time to enclose the target.

\section{Conclusions}

In this paper, a new implementation of a robot swarm for enclosing a target is examined. The swarm is equipped with the same limited transmission range signal emitter. Every robot is assigned a torch with the same specification and they can observe the sum of light intensity of their torches. The robot uses the direction with the strongest intensity of light which is an alternative to using the direction of its nearest neighbor agent. We proposed the scalable torch flashing strategy and the performance of them was evaluated. By the computer simulation, we confirmed that the proposed system can enclose a target.

\section{Reference}

1. T.H. Kim, T. Sugie, "Cooperative control for target capturing task based on a cyclic pursuit strategy", $\mathrm{Au}$ tomatica 43,1426-1431(2007)

2. Y. Kobayashi, K.Otsubo, S.Hosoe, "Autonomous De centralized Control of Capturing Behavior by Multiple Mobile Robots", Transactions of the Society of Instrument and Control Engineers, 43(8), 663671(2007)

3. M. Kubo, H. Sato, T.Yoshimura, A.Yamaguchi, T. Tanaka, "Multiple targets enclosure by robotic swarm", Robotics and Autonomous Systems 62,12941304(2014)

4. J. A. Marshall, M. E. Brouvke, B. A. Francis, "Formations of Vehicles in Cyclic Pursuit", IEEE Transactions on Automatic Contorol, 49, no.11, 1963-1974(2004)

5. I. Masubuchi, G. Zhai, "Control of Hybrid Systems-V : Analysis and Control of Switched Systems", Systems, Control and Information,52(1), 25-31(2008)

6. M.Rubenstein, C.Ahler, N.Hoff, A.Cabrera, R.Nagpal, "Kilobot: A low cost robot with scalable operations designed for collective behaviors", Robotics and Autonomous Systems, 62, 996-975,(2014).

7. Y. Takayama, S. Yamamoto, T. Takimoto, "Distributed Formation Control of a Nonholonomic Multiagent System for Target-enclosing Operations", Proceedings of 9th SICE System Integration Division Annual Conference, SY0015, 679-680(2008)

8. X. Xu, G. Zhai, "Some Results on Practical Asymptotic Stabilizability of Switched Systems", 44th IEEE Conference on Decision and Control 2005 and 2005 European Control Conference CDC-ECC '05,39984003(2005)

9. F. Ducatelle, A. Förster, G. D. Caro, and L. Gambardella, "New task allocation methods for robotic swarms", 9th IEEE/RAS Conference on Autonomous Robot Systems and Competitions,(2009). 